\title{
LOCALIZATION AND STRING DUALITY
}

\author{
KEFENG LIU
}

\section{INTRODUCTION}

According to string theorists, String Theory, as the most promising candidate for the grand unification of all fundamental forces in the nature, should be the final theory of the world, and should be unique. But now there are five different looking string theories. As argued by the physicists, these theories should be equivalent, in a way dual to each other. On the other hand all previous theories like the Yang-Mills and the Chern-Simons theory should be parts of string theory. In particular their partition functions should be equal or equivalent to each other in the sense that they are equal after certain transformation. To compute partition functions, physicists use localization technique, a modern version of residue theorem, on infinite dimensional spaces. More precisely they apply localization formally to path integrals which is not well-defined yet in mathematics. In many cases such computations reduce the path integrals to certain integrals of various Chern classes on various finite dimensional moduli spaces, such as the moduli spaces of stable maps and the moduli spaces of vector bundles. The identifications of these partition functions among different theories have produced many surprisingly beautiful mathematical formulas like the famous mirror formula [22], as well as the Mariño-Vafa formula $[25]$.

The mathematical proofs of these formulas from the string duality also depend on localization techniques on these various finite dimensional moduli spaces. The purpose of this note is to discuss our recent work on the subject. I will briefly discuss the proof of the Marinõ-Vafa formula, its generalizations and the related topological vertex theory [1]. More precisely we will use localization formulas in various form to compute the integrals of Chern classes on moduli spaces, and to prove those conjectures from string duality. For the proof of the Mariño-Vafa formula and the theory of topological vertex, we note that many aspects of mathematics are involved, such as the Chern-Simons knot invariants, combinatorics of symmetric groups, representations of Kac-Moody algebras, Calabi-Yau manifolds, geometry and topology of moduli space of stable maps, etc.

We remark that localization technique has been very successful in proving many conjectures from physics, see my ICM 2002 lecture [21] for more examples. One of our major tools in the proofs of these conjectures is the functorial localization formula which is a variation of the classical localization formula, it transfers computations on complicated spaces to simple spaces, and connects computations of mathematicians and physicists.

Starting from the proof of the Mariño-Vafa formula [18], we have proved a series of results about Hodge integrals on the moduli spaces of stable curves, GromovWitten invariants for open toric Calabi-Yau manifolds, and their relationship with 
equivariant indices of elliptic operators on the moduli spaces of framed stable bundles on the projective plane. Here we can only give a brief overview of the results and the main ideas of their proofs. For the details see [17], [15], [18], [19], [20], [13]. While the Marinõ-Vafa formula gives a close formula for the generating series of triple Hodge integrals on the moduli spaces of all genera and any number marked points, the mathematical theory of topological vertex [13] gives the most effective ways to compute the Gromov-Witten invariants of any open toric Calabi-Yau manifolds. Recently Pan Peng was able to use our results on topological vertex to give a complete proof of the Gopakumar-Vafa integrality conjecture for any open toric Calabi-Yau manifolds [27]. Kim also used our technique to derive new effective recursion formulas for Hodge integrals on the moduli spaces of stable curves [7].

The spirit of our results is the duality between gauge theory, Chern-Simons theory and the Calabi-Yau geometry in string theory. One of our observations about the geometric structure of the moduli spaces is the convolution formula which is encoded in the moduli spaces of relative stable maps [9], [10], and also in the combinatorics of symmetric groups, [18], [13]. This convolution structure implies the differential equation which we called the cut-and-join equation. The cut-and-join equation arises from both representation theory and geometry. The verification of the cut-and-join equation in combinatorics is a direct computation through character formulas, while its proof in geometry is quite subtle and involves careful analysis of the fixed points on the moduli spaces of relative stable maps, see [17]-[20] and [13] for more details. The coincidence of such kind of equation in both geometry and combinatorics is quite remarkable.

The mathematical theory of topological vertex was motivated by the physical theory as first developed by the Vafa group [1], who has been working on string duality for the past several years. Topological vertex theory is a high point of their work starting from their geometric engineering theory and Witten's conjecture that Chern-Simons theory is a string theory [29].

The Gopakumar-Vafa integrality conjecture is possibly the most interesting and challenging remaining conjecture in the subject of Gromov-Witten invariants. It is rather surprising that for some cases such invariants can be interpreted as the indices elliptic operators in gauge theory in [17]. A direct proof of the conjecture for open toric Calabi-Yau manifolds was given recently by Pan [27] by using the combinatorial formulas for the generating series of all genera and all degree GromovWitten invariants of open toric Calabi-Yau. These closed formulas are derived from the theory of topological vertex through the gluing property.

I will start with brief discussions about the Marinõ-Vafa conjecture, then about the other results mentioned above. This note is based on my lecture at the International Conference of Complex Geometry held at the Eastern China Normal University in the summer of 2004. I would like to thank the organizers for the hospitality during my visit. I would also like to thank my collaborators Melissa C.-C. Liu, Jian Zhou and Jun Li for the wonderful experience to solve these conjectures and to develop the theory together.

The great geometer Professor Shiing-Shen Chern passed away on December 3, 2004. I would like to dedicate this article to the memory of Professor Shiing-Shen Chern, from whom I learned not only mathematics, but also the philosophy of life. 


\section{The Mariño-VAFA Conjecture}

Our original motivation to study Hodge integrals was to find a general mirror formula for counting higher genus curves in Calabi-Yau manifolds. To generalize mirror principle to count the number of higher genus curves, we need to first compute Hodge integrals, i.e. the intersection numbers of the $\lambda$ classes and $\psi$ classes on the Deligne-Mumford moduli space of stable curves $\overline{\mathcal{M}}_{g, h}$. This moduli space is possibly the most famous and most interesting orbifold. It has been studied since Riemann, and by many Fields medalists for the past 50 years, from many different point of views. Still many interesting and challenging problems about the geometry and topology of these moduli spaces remain unsolved. String theory has motivated many fantastic conjectures about these moduli spaces including the famous Witten conjecture which is about the generating series of the integrals of the $\psi$-classes. We start with the introduction of some notations.

Recall that a point in $\overline{\mathcal{M}}_{g, h}$ consists of $\left(C, x_{1}, \ldots, x_{h}\right)$, a (nodal) curve $C$ of genus $g$, and $n$ distinguished smooth points on $C$. The Hodge bundle $\mathbb{E}$ is a rank $g$ vector bundle over $\overline{\mathcal{M}}_{g, h}$ whose fiber over $\left[\left(C, x_{1}, \ldots, x_{h}\right)\right]$ is $H^{0}\left(C, \omega_{C}\right)$, the complex vector space of holomorphic one forms on $C$. The $\lambda$ classes are the Chern Classes of $\mathbb{E}$,

$$
\lambda_{i}=c_{i}(\mathbb{E}) \in H^{2 i}\left(\overline{\mathcal{M}}_{g, h} ; \mathbb{Q}\right) .
$$

On the other hand, the cotangent line $T_{x_{i}}^{*} C$ of $C$ at the $i$-th marked point $x_{i}$ induces a line bundle $\mathbb{L}_{i}$ over $\overline{\mathcal{M}}_{g, h}$. The $\psi$ classes are the Chern classes:

$$
\psi_{i}=c_{1}\left(\mathbb{L}_{i}\right) \in H^{2}\left(\overline{\mathcal{M}}_{g, h} ; \mathbb{Q}\right) .
$$

Introduce the total Chern class

$$
\Lambda_{g}^{\vee}(u)=u^{g}-\lambda_{1} u^{g-1}+\cdots+(-1)^{g} \lambda_{g} .
$$

The Mariño-Vafa formula is about the generating series of the triple Hodge integrals

$$
\int_{\overline{\mathcal{M}}_{g, h}} \frac{\Lambda_{g}^{\vee}(1) \Lambda_{g}^{\vee}(\tau) \Lambda_{g}^{\vee}(-\tau-1)}{\prod_{i=1}^{h}\left(1-\mu_{i} \psi_{i}\right)}
$$

where $\tau$ is considered as a parameter here. Later we will see that it actually comes from the weight of the group action, and also from the framing of the knot. Taking Taylor expansions in $\tau$ or in $\mu_{i}$ one can obtain information on the integrals of the Hodge classes and the $\psi$-classes. The Marinõ-Vafa conjecture asserts that the generating series of such triple Hodge integrals for all genera and any numbers of marked points can be expressed by a close formula which is a finite expression in terms of representations of symmetric groups, or Chern-Simons knot invariants.

We remark that the moduli spaces of stable curves have been the sources of many interests from mathematics to physics. Mumford has computed some low genus numbers. The Witten conjecture, proved by Kontsevich, is about the integrals of the $\psi$-classes.

Let us briefly recall the background of the conjecture. Mariño and Vafa [25] made this conjecture based on the large $N$ duality between Chern-Simons and string theory. It starts from the conifold transition. We consider the resolution of singularity of the conifold $X$ defined by

$$
\left\{\left(\begin{array}{cc}
x & y \\
z & w
\end{array}\right) \in \mathbf{C}^{4}: x w-y z=0\right\}
$$


in two different ways:

(1). Deformed conifold $T^{*} S^{3}$

$$
\left\{\left(\begin{array}{cc}
x & y \\
z & w
\end{array}\right) \in \mathbf{C}^{4}: x w-y z=\epsilon\right\}
$$

where $\epsilon$ a real positive number. This is a symplectic resolution of the singularity.

(2). Resolved conifold by blowing up the singularity, which gives the total space

$$
\tilde{X}=\mathcal{O}(-1) \oplus \mathcal{O}(-1) \rightarrow \mathbf{P}^{1}
$$

which is explicitly given by

$$
\begin{aligned}
& \left\{\left(\left[Z_{0}, Z_{1}\right],\left(\begin{array}{cc}
x & y \\
z & w
\end{array}\right)\right) \in \mathbf{P}^{1} \times \mathbf{C}^{4}: \begin{array}{l}
(x, y) \in\left[Z_{0}, Z_{1}\right] \\
(z, w) \in\left[Z_{0}, Z_{1}\right]
\end{array}\right\} \\
& \begin{array}{ccc}
\tilde{X} & \subset & \mathbf{P}^{1} \times \mathbf{C}^{4} \\
\downarrow & & \downarrow \\
X & \subset & \mathbf{C}^{4}
\end{array}
\end{aligned}
$$

The brief history of the development of the conjecture is as follows. In 1992 Witten first conjectured that the open topological string theory on the deformed conifold $T^{*} S^{3}$ is equivalent to the Chern-Simons gauge theory on $S^{3}$. Such idea was pursued further by Gopakumar and Vafa in 1998, and then by Ooguri and Vafa in 2000. Based on the above conifold transition, they conjectured that the open topological string theory on the deformed conifold $T^{*} S^{3}$ is equivalent to the closed topological string theory on the resolved conifold $\tilde{X}$. Ooguri-Vafa only considered the zero framing case. Later Marinõ-Vafa generalized the idea to the non-zero framing case and discovered the beautiful formula for the generating series of the triple Hodge integrals. Recently Vafa and his collaborators systematically developed the theory, and for the past several years, they developed these duality ideas into the most effective tool to compute Gromov-Witten invariants on toric Calabi-Yau manifolds. The high point of their work is the theory of topological vertex. We refer to [25] and [1] for the details of the physical theory and the history of the development.

Starting with the proof of the Marinõ-Vafa conjecture [18], [19], we have developed a rather complete mathematical theory of topological vertex [13]. Many interesting consequences have been derived for the past year. Now let us see how the string theorists derived mathematical consequence from the above naive idea of string duality. First the Chern-Simons partition function has the form

$$
\langle Z(U, V)\rangle=\exp (-F(\lambda, t, V))
$$

where $U$ is the holonomy of the $U(N)$ Chern-Simons gauge field around the knot $K \subset S^{3}$, and $V$ is an extra $U(M)$ matrix. The partition function $\langle Z(U, V)\rangle$ gives the Chern-Simons knot invariants of $K$.

String duality asserts that the function $F(\lambda, t, V)$ should give the generating series of the open Gromov-Witten invariants of $\left(\tilde{X}, L_{K}\right)$, where $L_{K}$ is a Lagrangian submanifold of the resolved conifold $\tilde{X}$ canonically associated to the knot $K$. More precisely by applying the t'Hooft large $N$ expansion, and the "canonical" identifications of parameters similar to mirror formula, which at level $k$ are given by

$$
\lambda=\frac{2 \pi}{k+N}, \quad t=\frac{2 \pi i N}{k+N},
$$


we get the partition function of the topological string theory on conifold $\tilde{X}$, and then on $\mathbf{P}^{1}$. which is just the generating series of the Gromov-Witten invariants. This change of variables is very striking from the point of view of mathematics.

The special case when $K$ is the unknot is already very interesting. In non-zero framing it gives the Mariño-Vafa conjectural formula. In this case $\langle Z(U, V)\rangle$ was first computed in the zero framing by Ooguri-Vafa and in any framing $\tau \in \mathbb{Z}$ by Mariño-Vafa [25]. Comparing with Katz-Liu's computations of $F(\lambda, t, V)$, MariñoVafa conjectured the striking formula about the generating series of the triple Hodge integrals for all genera and any number of marked points in terms of the ChernSimons invariants, or equivalently in terms of the representations and combinatorics of symmetric groups. It is interesting to note that the framing in the MariñoVafa's computations corresponds to the choice of lifting of the circle action on the pair $\left(\tilde{X}, L_{\text {unknot }}\right)$ in Katz-Liu's localization computations. Both choices are parametrized by an integer $\tau$ which will be considered as a parameter in the triple Hodge integrals. Later we will take derivatives with respect to this parameter to get the cut-and-join equation.

It is natural to ask what mathematical consequence we can have for general duality, that is for general knots in general three manifolds, a first naive question is what kind of general Calabi-Yau manifolds will appear in the duality, in place of the conifold. Some special cases corresponding to the Seifert manifolds are known by gluing several copies of conifolds.

Now we give the precise statement of the Mariño-Vafa conjecture, which is an identity between the geometry of the moduli spaces of stable curves and ChernSimons knot invariants, or the combinatorics of the representation theory of symmetric groups.

Let us first introduce the geometric side. For every partition $\mu=\left(\mu_{1} \geq\right.$ $\left.\cdots \mu_{l(\mu)} \geq 0\right)$, we define the triple Hodge integral to be,

where the coefficient

$$
G_{g, \mu}(\tau)=A(\tau) \cdot \int_{\overline{\mathcal{M}}_{g, l(\mu)}} \frac{\Lambda_{g}^{\vee}(1) \Lambda_{g}^{\vee}(-\tau-1) \Lambda_{g}^{\vee}(\tau)}{\prod_{i=1}^{l(\mu)}\left(1-\mu_{i} \psi_{i}\right)},
$$

$$
A(\tau)=-\frac{\sqrt{-1}^{|\mu|+l(\mu)}}{|\operatorname{Aut}(\mu)|}[\tau(\tau+1)]^{l(\mu)-1} \prod_{i=1}^{l(\mu)} \frac{\prod_{a=1}^{\mu_{i}-1}\left(\mu_{i} \tau+a\right)}{\left(\mu_{i}-1\right) !} .
$$

The expressions, although very complicated, arise naturally from localization computations on the moduli spaces of relative stable maps into $\mathbf{P}^{1}$ with ramification type $\mu$ at $\infty$.

We now introduce the generating series

$$
G_{\mu}(\lambda ; \tau)=\sum_{g \geq 0} \lambda^{2 g-2+l(\mu)} G_{g, \mu}(\tau) .
$$

The special case when $g=0$ is given by

$$
\int_{\overline{\mathcal{M}}_{0, l(\mu)}} \frac{\Lambda_{0}^{\vee}(1) \Lambda_{0}^{\vee}(-\tau-1) \Lambda_{0}^{\vee}(\tau)}{\prod_{i=1}^{l(\mu)}\left(1-\mu_{i} \psi_{i}\right)}=\int_{\overline{\mathcal{M}}_{0, l(\mu)}} \frac{1}{\prod_{i=1}^{l(\mu)}\left(1-\mu_{i} \psi_{i}\right)}
$$

which is known to be equal to $|\mu|^{l(\mu)-3}$ for $l(\mu) \geq 3$, and we use this expression to extend the definition to the case $l(\mu)<3$. 
Introduce formal variables $p=\left(p_{1}, p_{2}, \ldots, p_{n}, \ldots\right)$, and define

$$
p_{\mu}=p_{\mu_{1}} \cdots p_{\mu_{l(\mu)}}
$$

for any partition $\mu$. These $p_{\mu_{j}}$ correspond to $\operatorname{Tr} V^{\mu_{j}}$ in the notations of string theorists. The generating series for all genera and all possible marked points are defined to be

$$
G(\lambda ; \tau ; p)=\sum_{|\mu| \geq 1} G_{\mu}(\lambda ; \tau) p_{\mu},
$$

which encode complete information of the triple Hodge integrals we are interested in.

Next we introduce the representation theoretical side. Let $\chi_{\mu}$ denote the character of the irreducible representation of the symmetric group $S_{|\mu|}$, indexed by $\mu$ with $|\mu|=\sum_{j} \mu_{j}$. Let $C(\mu)$ denote the conjugacy class of $S_{|\mu|}$ indexed by $\mu$. Introduce

$$
\mathcal{W}_{\mu}(\lambda)=\prod_{1 \leq a<b \leq l(\mu)} \frac{\sin \left[\left(\mu_{a}-\mu_{b}+b-a\right) \lambda / 2\right]}{\sin [(b-a) \lambda / 2]} \cdot \frac{1}{\prod_{i=1}^{l(\nu)} \prod_{v=1}^{\mu_{i}} 2 \sin [(v-i+l(\mu)) \lambda / 2]}
$$

This has an interpretation in terms of quantum dimension in Chern-Simons knot theory.

We define the following generating series

$$
R(\lambda ; \tau ; p)=\sum_{n \geq 1} \frac{(-1)^{n-1}}{n} \sum_{\mu}\left[\sum_{\cup_{i=1}^{n} \mu^{i}=\mu} \prod_{i=1}^{n} \sum_{\left|\nu^{i}\right|=\left|\mu^{i}\right|} \frac{\chi_{\nu^{i}}\left(C\left(\mu^{i}\right)\right)}{z_{\mu^{i}}} e^{\sqrt{-1}\left(\tau+\frac{1}{2}\right) \kappa_{\nu^{i}} \lambda / 2} \mathcal{W}_{\nu^{i}}(\lambda)\right] p_{\mu}
$$

where $\mu^{i}$ are sub-partitions of $\mu, z_{\mu}=\prod_{j} \mu_{j} ! j^{\mu_{j}}$ and

$$
\kappa_{\mu}=|\mu|+\sum_{i}\left(\mu_{i}^{2}-2 i \mu_{i}\right)
$$

for a partition $\mu$ which is also standard for representation theory of symmetric groups. There is the relation $z_{\mu}=|\operatorname{Aut}(\mu)| \mu_{1} \cdots \mu_{l(\mu)}$.

Finally we can give the precise statement of the Mariño-Vafa formula:

Conjecture: We have the identity

$$
G(\lambda ; \tau ; p)=R(\lambda ; \tau ; p)
$$

Before discussing the proof of this conjecture, we first give several remarks. This conjecture is a formula: $\mathrm{G}$ : Geometry $=\mathrm{R}$ : Representations, and the representations of symmetric groups are essentially combinatorics. We note that each $G_{\mu}(\lambda, \tau)$ is given by a finite and closed expression in terms of the representations of symmetric groups:

$$
G_{\mu}(\lambda, \tau)=\sum_{n \geq 1} \frac{(-1)^{n-1}}{n} \sum_{\cup_{i=1}^{n} \mu^{i}=\mu} \prod_{i=1}^{n} \sum_{\left|\nu^{i}\right|=\left|\mu^{i}\right|} \frac{\chi_{\nu^{i}}\left(C\left(\mu^{i}\right)\right)}{z_{\mu^{i}}} e^{\sqrt{-1}\left(\tau+\frac{1}{2}\right) \kappa_{\nu^{i}} \lambda / 2} \mathcal{W}_{\nu^{i}}(\lambda) .
$$

The generating series $G_{\mu}(\lambda, \tau)$ gives the values of the triple Hodge integrals for moduli spaces of curves of all genera with $l(\mu)$ marked points. Finally we remark that 
an equivalent expression of this formula is the following non-connected generating series. In this situation we have a relatively simpler combinatorial expression:

$$
G(\lambda ; \tau ; p)^{\bullet}=\exp [G(\lambda ; \tau ; p)]=\sum_{|\mu| \geq 0}\left[\sum_{|\nu|=|\mu|} \frac{\chi_{\nu}(C(\mu))}{z_{\mu}} e^{\sqrt{-1}\left(\tau+\frac{1}{2}\right) \kappa_{\nu} \lambda / 2} \mathcal{W}_{\nu}(\lambda)\right] p_{\mu}
$$

According to Mariño and Vafa, this formula gives values for all Hodge integrals up to three Hodge classes. This is almost right if we combine with some previously simple known formulas about Hodge integrals as proved by Lu.

By taking Taylor expansion in $\tau$ on both sides of the Mariño-Vafa formula, we have derived various Hodge integral identities in [20].

For example, as easy consequences of the Mariño-Vafa formula and the cut-andjoin equation as satisfied by the above generating series, we have unified simple proofs of the $\lambda_{g}$ conjecture by comparing the coefficients in $\tau$ in the Taylor expansions of the two expressions,

$$
\int_{\overline{\mathcal{M}}_{g, n}} \psi_{1}^{k_{1}} \cdots \psi_{n}^{k_{n}} \lambda_{g}=\left(\begin{array}{c}
2 g+n-3 \\
k_{1}, \ldots, k_{n}
\end{array}\right) \frac{2^{2 g-1}-1}{2^{2 g-1}} \frac{\left|B_{2 g}\right|}{(2 g) !},
$$

for $k_{1}+\cdots+k_{n}=2 g-3+n$, and the following identities for Hodge integrals:

$$
\int_{\overline{\mathcal{M}}_{g}} \lambda_{g-1}^{3}=\int_{\overline{\mathcal{M}}_{g}} \lambda_{g-2} \lambda_{g-1} \lambda_{g}=\frac{1}{2(2 g-2) !} \frac{\left|B_{2 g-2}\right|}{2 g-2} \frac{\left|B_{2 g}\right|}{2 g},
$$

where $B_{2 g}$ are Bernoulli numbers. And

$$
\int_{\overline{\mathcal{M}}_{g, 1}} \frac{\lambda_{g-1}}{1-\psi_{1}}=b_{g} \sum_{i=1}^{2 g-1} \frac{1}{i}-\frac{1}{2} \sum_{\substack{g_{1}+g_{2}=g \\ g_{1}, g_{2}>0}} \frac{\left(2 g_{1}-1\right) !\left(2 g_{2}-1\right) !}{(2 g-1) !} b_{g_{1}} b_{g_{2}}
$$

where

$$
b_{g}= \begin{cases}1, & g=0 \\ \frac{2^{2 g-1}-1}{2^{2 g-1}} \frac{\left|B_{2 g}\right|}{(2 g) !}, & g>0 .\end{cases}
$$

Now let us look at how we proved this conjecture. This is joint work with Chiu-Chu Liu, Jian Zhou.

The first proof of this formula is based on the Cut-and-Join equation which is a beautiful match of combinatorics and geometry. The details of the proof is given in [17] and [18]. First we look at the combinatorial side. Denote by $\left[s_{1}, \cdots, s_{k}\right]$ a $k$-cycle in the permutation group. We have the following two obvious operations:

Cut: a $k$-cycle is cut into an $i$-cycle and a $j$-cycle:

$$
[s, t] \cdot\left[s, s_{2}, \cdots, s_{i}, t, t_{2}, \cdots t_{j}\right]=\left[s, s_{2}, \cdots, s_{i}\right]\left[t, t_{2}, \cdots t_{j}\right] .
$$

Join: an $i$-cycle and a $j$-cycle are joined to an $(i+j)$-cycle:

$$
[s, t] \cdot\left[s, s_{2}, \cdots, s_{i}\right]\left[t, t_{2}, \cdots t_{j}\right]=\left[s, s_{2}, \cdots, s_{i}, t, t_{2}, \cdots t_{j}\right] .
$$

Such operations can be organized into differential equations which we call the cutand-join equation.

Now we look at the geometry side. In the moduli spaces of stable maps, cut and join have the following geometric meaning: Cut: one curve split into two lower 
degree or lower genus curves. Join: two curves joined together to give a higher genus or higher degree curve.

The combinatorics and geometry of cut-and-join are reflected in the following two differential equations, which look like heat equation. It is equivalent to a series of systems of linear ordinary differential equations. These equations are proved either by easy and direct computations in combinatorics or by localizations on moduli spaces of relative stable maps in geometry. In combinatorics, the proof is given by direct computations and was explored in the combinatorics in the mid $80 \mathrm{~s}$ and by Zhou [17] for this case. The differential operator on the right hand side corresponds to the cut-and-join operations which we also simply denote by $(C J)$.

\section{Proposition:}

$$
\frac{\partial R}{\partial \tau}=\frac{1}{2} \sqrt{-1} \lambda \sum_{i, j=1}^{\infty}\left((i+j) p_{i} p_{j} \frac{\partial R}{\partial p_{i+j}}+i j p_{i+j}\left(\frac{\partial R}{\partial p_{i}} \frac{\partial R}{\partial p_{j}}+\frac{\partial^{2} R}{\partial p_{i} \partial p_{j}}\right)\right) .
$$

On the geometry side the proof of such equation is given by localization on the moduli spaces of relative stable maps into the the projective line $\mathbf{P}^{1}$ with fixed ramifications at $\infty$ :

\section{Proposition:}

$$
\frac{\partial G}{\partial \tau}=\frac{1}{2} \sqrt{-1} \lambda \sum_{i, j=1}^{\infty}\left((i+j) p_{i} p_{j} \frac{\partial G}{\partial p_{i+j}}+i j p_{i+j}\left(\frac{\partial G}{\partial p_{i}} \frac{\partial G}{\partial p_{j}}+\frac{\partial^{2} G}{\partial p_{i} \partial p_{j}}\right)\right) .
$$

The proof of the above equation is given in [17]

Initial Value: $\tau=0$,

$$
G(\lambda, 0, p)=\sum_{d=1}^{\infty} \frac{p_{d}}{2 d \sin \left(\frac{\lambda d}{2}\right)}=R(\lambda, 0, p) .
$$

which is precisely the Ooguri-Vafa formula and which has been proved previously [30]. Since the solution is unique, we therefore obtain the equality which is the Mariño-Vafa conjecture:

Theorem: We have the identity

$$
G(\lambda ; \tau ; p)=R(\lambda ; \tau ; p) .
$$

During the proof we note that the cut-and-join equation is encoded in the geometry of the moduli spaces of stable maps. In fact we later find the convolution formula of the following form, which is a relation for the disconnected version $G^{\bullet}=\exp G$,

$$
G_{\mu}^{\bullet}(\lambda, \tau)=\sum_{|\nu|=|\mu|} \Phi_{\mu, \nu}^{\bullet}(-\sqrt{-1} \tau \lambda) z_{\nu} K_{\nu}^{\bullet}(\lambda)
$$

where $\Phi_{\mu, \nu}^{\bullet}$ is the generating series of double Hurwitz numbers, and $z_{\nu}$ is the combinatorial constant appeared in the previous formulas. Equivalently this gives the explicit solution of the cut-and-join differential equation with initial value $K^{\bullet}(\lambda)$, which is the generating series of the integrals of certain Euler classes on the moduli spaces of relative stable maps to $\mathbf{P}^{1}$. See [14] for the derivation of this formula, and see [19] for the two partition analogue. 
The Witten conjecture as proved by Kontsevich states that the generating series of the $\psi$-class integrals satisfy infinite number of differential equations. The remarkable point of Mariño-Vafa formula is that it gives a finite close formula. In fact by taking limits in $\tau$ and $\mu_{i}$ 's one can obtain the Witten conjecture as argued by Okounkov-Pandhrapande. But the combinatorics involved is non-trivial.

The same argument as our proof of the conjecture gives a simple and geometric proof of the ELSV formula for Hurwitz numbers. It reduces to the fact that the push-forward of 1 is equal to 1 in equivariant cohomology for a generically one-toone map. See [18] for more details.

We would like to briefly explain the technical details of the proof. The proof of the combinatorial cut-and-join formula is based on the Burnside formula and various simple results in symmetric functions. See [30], [15] and [18].

The proof of the geometric cut-and-join formula used the functorial localization formula in [22] and [23]. Here we only state its simple form for manifolds as used in [22], the virtual version of this formula is proved and used in [23].

Given $X$ and $Y$ two compact manifolds with torus action. Let $f: X \rightarrow Y$ be an equivariant map. Let $F \subset Y$ be a fixed component, and let $E \subset f^{-1}(F)$ denote the fixed components lying inside $f^{-1}(F)$. Let $f_{0}=\left.f\right|_{E}$, then we have

Functorial Localization Formula: For $\omega \in H_{T}^{*}(X)$ an equivariant cohomology class, we have the identity on F:

$$
f_{0 *}\left[\frac{i_{E}^{*} \omega}{e_{T}(E / X)}\right]=\frac{i_{F}^{*}\left(f_{*} \omega\right)}{e_{T}(F / Y)} .
$$

This formula, which is a generalization of the Atiyah-Bott localization formula to relative setting, has been applied to various settings to prove many interesting conjectures from physics. It was discovered and effectively used in [22]. A virtual version which was first applied to the virtual fundamental cycles in the computations of Gromov-Witten invariants was first proved and used in [23].

This formula is very effective and useful because we can use it to push computations on complicated moduli space to simpler moduli space. The moduli spaces used by mathematicians are usually the correct but complicated moduli spaces like the moduli spaces of stable maps, while the moduli spaces used by physicists are usually the simple but the wrong ones like the projective spaces. This functorial localization formula has been used successfully in the proof of the mirror formula [22], [23], the proof of the Hori-Vafa formula [16], and the easy proof of the ELSV formula [18]. Our first proof of the Mariño-Vafa formula also used this formula in a crucial way.

More precisely, let $\mathcal{M}_{g}\left(\mathbf{P}^{1}, \mu\right)$ denote the moduli space of relative stable maps from a genus $g$ curve to $\mathbf{P}^{1}$ with fixed ramification type $\mu$ at $\infty$, where $\mu$ is a fixed partition. We apply the functorial localization formula to the divisor morphism from the relative stable map moduli space to the projective space,

$$
\mathrm{Br}: \mathcal{M}_{g}\left(\mathbf{P}^{1}, \mu\right) \rightarrow \mathbf{P}^{r},
$$

where $r$ denotes the dimension of $\mathcal{M}_{g}\left(\mathbf{P}^{1}, \mu\right)$. This is similar to the set-up of mirror principle, only with a different linearized moduli space, but in both cases the target spaces are projective spaces.

We found that the fixed points of the target $\mathbf{P}^{r}$ precisely labels the cut-andjoin operations of the triple Hodge integrals. Functorial localization reduces the 
problem to the study of polynomials in the equivariant cohomology group of $\mathbf{P}^{r}$. We were able to squeeze out a system of linear equations which implies the cutand-join equation. Actually we derived a stronger relation than the cut-and-join equation, while the cut-and-join equation we need for the Mariño-Vafa formula is only the very first of such kind of relations. See [18] for higher order cut-and-join equations.

As was known in infinite Lie algebra theory, the cut-and-join operator is closely related to and more fundamental than the Virasoro algebras in some sense.

Recently there have appeared two different approaches to the Mariño-Vafa formula. The first one is a direct derivation of the convolution formula which was discovered during our proof of the two partition analogue of the formula [19]. See [14] for the details of the derivation in this case. The second is by OkounkovPandhrapande [26], they gave a different approach by using the ELSV formula as initial value, and as well as the $\lambda_{g}$ conjecture and other recursion relations from localization on the moduli spaces of stable maps to $\mathbf{P}^{1}$.

\section{Two Partition Formula}

The two partition analogue of the Mariño-Vafa formula naturally arises from the localization computations of the Gromov-Witten invariants of the open toric Calabi-Yau manifolds, as explained in [31].

To state the formula we let $\mu^{+}, \mu^{-}$be any two partitions. Introduce the Hodge integrals involving these two partitions:

$$
G_{\mu^{+}, \mu^{-}}(\lambda ; \tau)=B\left(\tau ; \mu^{+}, \mu^{-}\right) \cdot \sum_{g \geq 0} \lambda^{2 g-2} A_{g}\left(\tau ; \mu^{+}, \mu^{-}\right)
$$

where

$$
A_{g}\left(\tau ; \mu^{+}, \mu^{-}\right)=\int_{\overline{\mathcal{M}}_{g, l\left(\mu^{+}\right)+l\left(\mu^{-}\right)}} \frac{\Lambda_{g}^{\vee}(1) \Lambda_{g}^{\vee}(\tau) \Lambda_{g}^{\vee}(-\tau-1)}{\prod_{i=1}^{l\left(\mu^{+}\right)}\left(1-\mu_{i}^{+} \psi_{i}\right) \prod_{j=1}^{l\left(\mu^{-}\right)} \tau\left(\tau-\mu_{i}^{-} \psi_{j+l\left(\mu^{+}\right)}\right)}
$$

and

$$
\begin{gathered}
B\left(\tau ; \mu^{+}, \mu^{-}\right)=-\frac{(\sqrt{-1} \lambda)^{l\left(\mu^{+}\right)+l\left(\mu^{-}\right)}}{\left|\operatorname{Aut}\left(\mu^{+}\right)\right|\left|\operatorname{Aut}\left(\mu^{-}\right)\right|}[\tau(\tau+1)]^{l\left(\mu^{+}\right)+l\left(\mu^{-}\right)-1} . \\
\prod_{i=1}^{l\left(\mu^{+}\right)} \frac{\prod_{a=1}^{\mu_{i}^{+}-1}\left(\mu_{i}^{+} \tau+a\right)}{\left(\mu_{i}^{+}-1\right) !} \cdot \prod_{i=1}^{l\left(\mu^{-}\right)} \frac{\prod_{a=1}^{\mu_{i}^{-}-1}\left(\mu_{i}^{-} \frac{1}{\tau}+a\right)}{\left(\mu_{i}^{-}-1\right) !} .
\end{gathered}
$$

These complicated expressions naturally arise in open string theory, as well as in the localization computations of the Gromov-Witten invariants on open toric Calabi-Yau manifolds.

We introduce two generating series, first on the geometry side,

$$
G^{\bullet}\left(\lambda ; p^{+}, p^{-} ; \tau\right)=\exp \left(\sum_{\left(\mu^{+}, \mu^{-}\right) \in \mathcal{P}^{2}} G_{\mu^{+}, \mu^{-}}(\lambda, \tau) p_{\mu^{+}}^{+} p_{\mu^{-}}^{-}\right),
$$

where $p_{\mu^{ \pm}}^{ \pm}$are two sets of formal variables associated to the two partitions.

On the representation side, we introduce 
$R^{\bullet}\left(\lambda ; p^{+}, p^{-} ; \tau\right)=\sum_{\left|\nu^{ \pm}\right|=\left|\mu^{ \pm}\right| \geq 0} \frac{\chi_{\nu^{+}}\left(C\left(\mu^{+}\right)\right)}{z_{\mu^{+}}} \frac{\chi_{\nu^{-}}\left(C\left(\mu^{-}\right)\right)}{z_{\mu^{-}}} \cdot e^{\sqrt{-1}\left(\kappa_{\nu}+\tau+\kappa_{\nu^{-}} \tau^{-1}\right) \lambda / 2} \mathcal{W}_{\nu^{+}, \nu^{-}} p_{\mu^{+}}^{+} p_{\mu^{-}}^{-}$.

Here

$$
\begin{gathered}
\mathcal{W}_{\mu, \nu}=q^{l(\nu) / 2} \mathcal{W}_{\mu} \cdot s_{\nu}\left(\mathcal{E}_{\mu}(t)\right) \\
=(-1)^{|\mu|+|\nu|} q^{\frac{\kappa_{\mu}+\kappa_{\nu}+|\mu|+|\nu|}{2}} \sum_{\rho} q^{-|\rho|} s_{\mu / \rho}(1, q, \ldots) s_{\nu / \rho}(1, q, \ldots)
\end{gathered}
$$

in terms of the skew Schur functions $s_{\mu}$ [24]. They appear naturally in the ChernSimons invariant of the Hopf link.

Theorem: We have the identity:

$$
G^{\bullet}\left(\lambda ; p^{+}, p^{-} ; \tau\right)=R^{\bullet}\left(\lambda ; p^{+}, p^{-} ; \tau\right) .
$$

The idea of the proof is similar to that of the proof of the Mariño-Vafa formula. We prove that both sides of the above identity satisfy the same cut-and-join equation of the following type:

$$
\frac{\partial}{\partial \tau} H^{\bullet}=\frac{1}{2}(C J)^{+} H^{\bullet}-\frac{1}{2 \tau^{2}}(C J)^{-} H^{\bullet},
$$

where $(C J)^{ \pm}$cut-and-join operator, the differential operator with respect to the two set of variables $p^{ \pm}$. We then prove that they have the same initial value at $\tau=-1$ :

$$
G^{\bullet}\left(\lambda ; p^{+}, p^{-} ;-1\right)=R^{\bullet}\left(\lambda ; p^{+}, p^{-} ;-1\right),
$$

which is again given by the Ooguri-Vafa formula [19], [31].

The cut-and-join equation can be written in a linear matrix form, and such equation follows from the convolution formula of the form

$$
K_{\mu^{+}, \mu^{-}}^{\bullet}(\lambda)=\sum_{\left|\nu^{ \pm}\right|=\mu^{ \pm}} G_{\mu^{+}, \mu^{-}}^{\bullet}(\lambda ; \tau) z_{\nu^{+}} \Phi_{\nu^{+}, \mu^{+}}^{\bullet}(-\sqrt{-1} \lambda \tau) z_{\nu^{-}} \Phi_{\nu^{-}, \mu^{-}}^{\bullet}\left(\frac{-\sqrt{-1}}{\tau} \lambda\right)
$$

where $\Phi^{\bullet}$ denotes the generating series of double Hurwitz numbers, and $K_{\mu^{+}, \mu^{-}}$ is the generating series of certain integrals on the moduli spaces of relative stable maps. For more details see [19].

This convolution formula arises naturally from localization computations on the moduli spaces of relative stable maps to $\mathbf{P}^{1} \times \mathbf{P}^{1}$ with the point $(\infty, \infty)$ blown up. So it reflects the geometric structure of the moduli spaces. Such convolution type formula was actually discovered during our search for a proof of this formula, both on the geometric and the combinatorial side, see [19] for the detailed derivations of the convolution formulas in both geometry and combinatorics.

The proof of the combinatorial side of the convolution formula is again a direct computation. The proof of the geometric side for the convolution equation is to reorganize the generating series from localization contributions on the moduli spaces of relative stable maps into $\mathbf{P}^{1} \times \mathbf{P}^{1}$ with the point $(\infty, \infty)$ blown up, in terms of the double Hurwitz numbers. It involves careful analysis and computations. 


\section{Three Partition Formula}

When we worked on the Mariño-Vafa formula and its generalizations, we were simply trying to generalize the method and the formula to involve more partitions, but it turned out that in the three partition case, we naturally met the theory of topological vertex. Topological vertex was first introduced in string theory by Vafa et al in [1], it can be deduced from a three partition analogue of the MariñoVafa formula in a highly nontrivial way. From this we were able to give a rigorous mathematical foundation for the physical theory. Topological vertex is a high point of the theory of string duality as developed by Vafa and his group for the past several years, starting from Witten's conjectural duality between Chern-Simons and open string theory. It gives the most powerful and effective way to compute the Gromov-Witten invariants for all open toric Calabi-Yau manifolds. In physics it is rare to have two theories agree up to all orders. In mathematics the theory of topological vertex already has many interesting applications. Here we only briefly sketch the rough idea for the three partition analogue of the Mariño-Vafa formula. For its relation to the theory of topological vertex, we refer the reader to [13] for the details.

Given any three partitions $\vec{\mu}=\left\{\mu^{1}, \mu^{2}, \mu^{3}\right\}$, the cut-and-join equation in this case, for both the geometry and representation sides, has the form:

$\frac{\partial}{\partial \tau} F^{\bullet}(\lambda ; \tau ; \mathbf{p})=(C J)^{1} F^{\bullet}(\lambda ; \tau ; \mathbf{p})+\frac{1}{\tau^{2}}(C J)^{2} F^{\bullet}(\lambda ; \tau ; \mathbf{p})+\frac{1}{(\tau+1)^{2}}(C J)^{3} F^{\bullet}(\lambda ; \tau ; \mathbf{p})$.

The cut-and-join operators $(C J)^{1},(C J)^{2}$ and $(C J)^{3}$ are with respect to the three partitions. More precisely they correspond to the differential operators with respect to the three groups of infinite numbers of variables $\mathbf{p}=\left\{p^{1}, p^{2}, p^{3}\right\}$.

The initial value for this differential equation is taken at $\tau=1$, which is then reduced to the formulas of two partition case. The combinatorial, or the ChernSimons invariant side is given by $\mathcal{W}_{\vec{\mu}}=\mathcal{W}_{\mu^{1}, \mu^{2}, \mu^{3}}$ which is a combination of the $\mathcal{W}_{\mu, \nu}$ as in the two partition case. See [13] for its explicit expression.

On the geometry side,

$$
G^{\bullet}(\lambda ; \tau ; \mathbf{p})=\exp (G(\lambda ; \tau ; \mathbf{p}))
$$

is the non-connected version of the generating series of the triple Hodge integral. More precisely,

$$
G(\lambda ; \tau ; \mathbf{p})=\sum_{\vec{\mu}}\left[\sum_{g=0}^{\infty} \lambda^{2 g-2+l(\vec{\mu})} G_{g, \vec{\mu}}(\tau)\right] p_{\mu^{1}}^{1} p_{\mu^{2}}^{2} p_{\mu_{3}}^{3}
$$

where $l(\vec{\mu})=l\left(\mu^{1}\right)+l\left(\mu^{2}\right)+l\left(\mu^{3}\right)$ and $G_{g, \vec{\mu}}(\tau)$ denotes the Hodge integrals of the following form,

$A(\tau) \int_{\overline{\mathcal{M}}_{g, l_{1}+l_{2}+l_{3}}} \frac{\Lambda_{g}^{\vee}(1) \Lambda_{g}^{\vee}(\tau) \Lambda_{g}^{\vee}(-\tau-1)}{\prod_{j=1}^{l_{1}}\left(1-\mu_{j}^{1} \psi_{j}\right) \prod_{j=1}^{l_{2}} \tau\left(\tau-\mu_{j}^{2} \psi_{l_{1}+j}\right)} \cdot \frac{(\tau(\tau+1))^{l_{1}+l_{2}+l_{3}-1}}{\prod_{j=1}^{l_{3}}(\tau+1)\left(\tau+1+\mu_{j}^{3} \psi_{l_{1}+l_{2}+j}\right)}$,

where

$$
A(\tau)=\frac{-(\sqrt{-1} \lambda)^{l_{1}+l_{2}+l_{3}}}{\left|\operatorname{Aut}\left(\mu^{1}\right) \| \operatorname{Aut}\left(\mu^{2}\right)\right|\left|\operatorname{Aut}\left(\mu^{3}\right)\right|} \prod_{j=1}^{l_{1}} \frac{\prod_{a=1}^{\mu_{j}^{1}-1}\left(\tau \mu_{j}^{1}+a\right)}{\left(\mu_{j}^{1}-1\right) !} .
$$




$$
\prod_{j=1}^{l_{2}} \frac{\prod_{a=1}^{\mu_{j}^{1}-1}\left((-1-1 / \tau) \mu_{j}^{2}+a\right)}{\left(\mu_{j}^{2}-1\right) !} \prod_{j=1}^{l_{3}} \frac{\prod_{a=1}^{\mu_{1}^{1}-1}\left(-\mu_{j}^{3} /(\tau+1)+a\right)}{\left(\mu_{j}^{3}-1\right) !}
$$

In the above expression, $l_{i}=l\left(\mu^{i}\right), i=1,2,3$. Although its complicated coefficients, these triple integrals naturally arise from localizations on the moduli spaces of relative stable maps into the blow-up of $\mathbf{P}^{1} \times \mathbf{P}^{1} \times \mathbf{P}^{1}$ along certain divisors. It also naturally appears in open string theory computations [1]. See [13] for more details.

One of our results in [13] states that $G^{\bullet}(\lambda ; \tau ; \mathbf{p})$ has a combinatorial expression $R^{\bullet}(\lambda ; \tau ; \mathbf{p})$ in terms of the Chern-Simons knot invariants $W_{\vec{\mu}}$, which is a closed combinatorial expression. More precisely it is given by

$$
R^{\bullet}(\lambda ; \tau ; \mathbf{p})=\sum_{\vec{\mu}}\left[\sum_{\left|\nu^{i}\right|=\left|\mu^{i}\right|} \prod_{i=1}^{3} \frac{\chi_{\nu^{i}}\left(\mu^{i}\right)}{z_{\mu^{i}}} q^{\frac{1}{2}\left(\sum_{i=1}^{3} \kappa_{\nu^{i}} \frac{w_{i+1}}{w_{i}}\right)} W_{\vec{\nu}}(q)\right] p_{\mu^{1}}^{1} p_{\mu^{2}}^{2} p_{\mu^{3}}^{3} .
$$

Here $w_{4}=w_{1}$ and $w_{3}=-w_{1}-w_{2}$ and $\tau=\frac{w_{2}}{w_{1}}$. Due to the complicated combinatorics in the initial values, the combinatorial expression $W_{\vec{\mu}}$ we obtained is different from the expression $\mathcal{W}_{\vec{\mu}}$ obtained by Vafa et al. Actually our expression is even simpler than theirs in some sense. The expression we obtained is more convenient for mathematical applications such as the proof of the Gopakumar-Vafa conjecture for open toric Calabi-Yau manifolds. It should be possible to identify the two combinatorial expressions by using the classical theory of symmetric functions, as pointed out to us by R. Stanley.

Theorem: We have the equality:

$$
G^{\bullet}(\lambda ; \tau ; \mathbf{p})=R^{\bullet}(\lambda ; \tau ; \mathbf{p})
$$

The key point to prove the above theorem is still the proof of convolution formulas for both sides which imply the cut-and-join equation. The proof of the convolution formula for $G^{\bullet}(\lambda ; \tau ; \mathbf{p})$ is much more complicated than the one and two partition cases. See [13] for details.

The most useful property of topological vertex is its gluing property induced by the orthogonal relations of the characters of the symmetric group. This is very close to the situation of two dimensional gauge theory. In fact string theorists consider topological vertex as a kind of lattice theory on Calabi-Yau manifolds. By using the gluing formula we can easily obtain closed formulas for generating series of Gromov-Witten invariants of all genera and all degrees, open or closed, for all open toric Calabi-Yau manifolds, in terms of the Chern-Simons knot invariants. Such formulas are always given by finite sum of products of those Chern-Simons type invariants $\mathcal{W}_{\mu, \nu}$ 's. The magic of topological vertex is that, by simply looking at the moment map graph of the toric surfaces in the open toric Calabi-Yau, we can immediately write down the closed formula for the generating series for all genera and all degree Gromov-Witten invariants, or more precisely the Euler numbers of certain bundles on the moduli space of stable maps.

Here we only give one example to describe the topological vertex formula for the generating series of the all degree and all genera Gromov-Witten invariants 
for the open toric Calabi-Yau 3-fold $O(-3) \longrightarrow \mathbf{P}^{2}$ in terms of the Chern-Simons invariants. In this case the formula is given by

$\exp \left(\sum_{g=0}^{\infty} \lambda^{2 g-2} F_{g}(t)\right)=\sum_{\nu_{1}, \nu_{2}, \nu_{3}} \mathcal{W}_{\nu_{1}, \nu_{2}} \mathcal{W}_{\nu_{2}, \nu_{3}} \mathcal{W}_{\nu_{3}, \nu_{1}}(-1)^{\sum_{j=1}^{3}\left|\nu_{j}\right|} q^{\frac{1}{2} \sum_{i=1}^{3} \kappa_{\nu_{i}}} e^{t\left(\sum_{j=1}^{3}\left|\nu_{j}\right|\right)}$

where $q=e^{\sqrt{-1} \lambda}$. The precise definition of $F_{g}(t)$ will be given in the next section.

For general open toric Calabi-Yau manifolds, the expressions are just similar. They are all given by finite and closed formulas, which are easily read out from the moment map graphs associated to the toric surfaces, with the topological vertex associated to each vertex of the graph.

In [1] Vafa and his group first developed the theory of topological vertex by using string duality between Chern-Simons and Calabi-Yau, which is a physical theory. In [13] we established the mathematical theory of the topological vertex, and derived various mathematical corollaries, including the relation of the GromovWitten invariants to the equivariant index theory as motivated by the Nekrasov conjecture in string duality [17].

\section{Gopakumar-Vafa Conjecture and Equivariant Indices of Elliptic OPERATORS}

Let $N_{g, d}$ denote the so-called Gromov-Witten invariant of genus $g$ and degree $d$ of an open toric Calabi-Yau 3-fold. $N_{g, d}$ is defined to be the Euler number of the obstruction bundle on the moduli space of stable maps of degree $d \in H_{2}(S, \mathbb{Z})$ from genus $g$ curve into the surface base $S$. The open toric Calabi-Yau manifold associated to the toric surface $S$ is the total space of the canonical line bundle $K_{S}$ on $S$. More precisely

$$
N_{g, d}=\int_{\left[\overline{\mathcal{M}}_{g}(S, d)\right]^{v}} e\left(V_{g, d}\right)
$$

with $V_{g, d}=R^{1} \pi_{*} u^{*} K_{S}$ a vector bundle on the moduli induced by the canonical bundle $K_{S}$. Here $\pi: U \rightarrow \overline{\mathcal{M}}_{g}(S, d)$ denotes the universal curve and $u$ can be considered as the evaluation or universal map. Let us write

$$
F_{g}(t)=\sum_{d \geq 0} N_{g, d} e^{-d \cdot t}
$$

The Gopakumar-Vafa conjecture is stated as follows:

Gopakumar-Vafa Conjecture: There exists an expression:

$$
\sum_{g=0}^{\infty} \lambda^{2 g-2} F_{g}(t)=\sum_{k=1}^{\infty} \sum_{g, d \geq 0} n_{d}^{g} \frac{1}{d}\left(2 \sin \frac{d \lambda}{2}\right)^{2 g-2} e^{-k d \cdot t}
$$

such that $n_{d}^{g}$ are integers, called instanton numbers.

Motivated by the Nekrasov duality conjecture between the four dimensional gauge theory and string theory, we are able to interpret the above integers $n_{d}^{g}$ 
as equivariant indices of certain elliptic operators on the moduli spaces of anti-selfdual connections [17]:

Theorem: For certain interesting cases, these $n_{d}^{g}$ 's can be written as equivariant indices on the moduli spaces of anti-self-dual connections on $\mathbb{C}^{2}$.

For more precise statement, we refer the reader to [17]. The interesting cases include open toric Calabi-Yau manifolds when $S$ is Hirzebruch surface. The proof of this theorem is to compare fixed point formula expressions for equivariant indices of certain elliptic operators on the moduli spaces of anti-self-dual connections with the combinatorial expressions of the generating series of the Gromov-Witten invariants on the moduli spaces of stable maps. They both can be expressed in terms of Young diagrams of partitions. We find that they agree up to certain highly nontrivial "mirror transformation", a complicated variable change. This result is not only interesting for the index formula interpretation of the instanton numbers, but also for the fact that it gives the first complete examples that the Gopakumar-Vafa conjecture holds for all genera and all degrees.

Recently P. Pan [27] has given a proof of the Gopakumar-Vafa conjecture for all open toric Calabi-Yau 3-folds by using the Chern-Simons expressions from the topological vertex. His method is to explore the property of the Chern-Simons expression in great detail with some clever observation about the form of the combinatorial expressions. On the other hand, Kim in [7] has derived some remarkable recursion formulas for Hodge integrals of all genera and any number of marked points, involving one $\lambda$-classes. His method is to add marked points in the moduli spaces and then follow the localization argument we used to prove the Mariño-Vafa formula.

We strongly believe that there is a more interesting and grand duality picture between Chern-Simons invariants for three dimensional manifolds and the GromovWitten invariants for open toric Calabi-Yau manifolds. Our proofs of the MariñoVafa formula, and the setup of the mathematical foundation for topological vertex theory and the results of Pan and Kim all together have just opened a window for a more splendid picture.

\section{REFERENCES}

[1] M. Aganagic, A. Klemm, M. Marino, C. Vafa, The topological vertex, preprint, hepth/0305132.

[2] M. Aganagic, M. Marino, C. Vafa, All loop topological string amplitudes from Chern-Simons theory, preprint, hep-th/0206164.

[3] T. Ekedahl, S. Lando, M. Shapiro, A. Vainshtein, Hurwitz numbers and intersections on moduli spaces of curves. Invent. Math. 146 (2001), no. 2, 297-327.

[4] I.P. Goulden, D.M. Jackson, A. Vainshtein, The number of ramified coverings of the sphere by the torus and surfaces of higher genera, Ann. of Comb. 4 (2000), 27-46.

[5] A. Iqbal, All genus topological amplitudes and 5-brane webs as Feynman diagrams, preprint, hep-th/0207114.

[6] S. Katz, C.-C. Liu, Enumerative geometry of stable maps with Lagrangian boundary conditions and multiple covers of the disc, Adv. Theor. Math. Phys. 5 (2001), 1-49.

[7] Y. Kim, Computing Hodge integrals with one lambda-class, preprint, math-ph/0501018.

[8] M. Kontsevich, Intersection theory on the moduli space of curves and the matrix Airy function. Comm. Math. Phys. 147 (1992), no. 1, 1-23.

[9] J. Li, Stable Morphisms to singular schemes and relative stable morphisms, J. Diff. Geom. 57 (2001), 509-578. 
[10] J. Li, Relative Gromov-Witten invariants and a degeneration formula of Gromov-Witten invariants, J. Diff. Geom. 60 (2002), 199-293.

[11] J. Li, Lecture notes on relative $G W$-invariants, preprint.

[12] A.M. Li, G. Zhao, Q. Zheng, The number of ramified coverings of a Riemann surface by Riemann surface, Comm. Math. Phys. 213 (2000), no. 3, 685-696.

[13] J. Li, C.-C. Liu, K. Liu, J. Zhou, A mathematical theory of the topological vertex, preprint, math.AG/0411247.

[14] C.-C. Liu, Formulae of one-partition and two-partition Hodge integrals, preprint, math.AG/0502430.

[15] J. Li, K. Liu, J. Zhou, Topological string partition functions as equivariant indices, preprint, math.AG/0412089.

[16] B. Lian, C.-H. Liu, K. Liu, S.-T. Yau, The $S^{1}$-fixed points in quot-schemes and mirror principle computations, Contemp. Math., 322, 2003, 165-194.

[17] C.-C. Liu, K. Liu, J. Zhou, On a proof of a conjecture of Mariño-Vafa on Hodge Integrals, Math. Res. Letters 11, 259-272(2004).

[18] C.-C. Liu, K. Liu, J. Zhou, A proof of a conjecture of Mariño-Vafa on Hodge Integrals, J. Differential Geometry, 65(2003) 289-340.

[19] C.-C, Liu, K. Liu, J. Zhou, A formula of two-partition Hodge integrals, preprint, math.AG/0310272.

[20] C.-C, Liu, K. Liu, J. Zhou, Mariño-Vafa formula and Hodge integral identities, preprint, math.AG/0308015.

[21] K. Liu, Mathematical results inspired by physics, Proc. ICM 2002, Vol. III, 457-466.

[22] B. Lian, K. Liu, S.-T. Yau, Mirror Principle I, Asian J. Math., 1, 1997, 729-763.

[23] B. Lian, K. Liu, S.-T. Yau, Mirror Principle III, Asian J. Math., 3, 1999, 771-800.

[24] I.G. MacDonald, Symmetric functions and Hall polynomials, 2nd edition.Claredon Press, 1995.

[25] M. Mariño, C. Vafa, Framed knots at large $N$, Orbifolds in mathematics and physics (Madison, WI, 2001), 185-204, Contemp. Math., 310, Amer. Math. Soc., Providence, RI, 2002.

[26] A. Okounkov, R. Pandharipande, Hodge integrals and invariants of the unknots, preprint, math.AG/0307209.

[27] P. Pan, A simple proof of Gopakumar-Vafa conjecture for local toric Calabi-Yau manifolds, preprint, math.AG/0410540.

[28] E. Witten, Quantum field theory and the Jones polynomial, Commun. Math. Phys. 121 (1989) 351-399.

[29] E. Witten, Two-dimensional gravity and intersection theory on moduli space. Surveys in differential geometry (Cambridge, MA, 1990), 243-310, Lehigh Univ., Bethlehem, PA, 1991.

[30] J. Zhou, Hodge integrals, Hurwitz numbers, and symmetric groups, preprint, math.AG/0308024.

[31] J. Zhou, A conjecture on Hodge integrals, preprint.

[32] J. Zhou, Localizations on moduli spaces and free field realizations of Feynman rules, preprint.

Center of Mathematical Sciences, Zhejiang University, and Department of MatheMATICS, UCLA

E-mail address: liu@math.ucla.edu 